\title{
Influence of casting defects on fatigue strength of an investment cast Ti-6Al-4V alloy
}

\author{
Gaëlle Léopold ${ }^{1}$, Yves Nadot ${ }^{1}$, José Mendez ${ }^{1}$ and Thomas Billaudeau ${ }^{2}$ \\ ${ }^{1}$ Institut Pprime, CNRS UPR 3346, ISAE-ENSMA, Université de Poitiers, Département Physique et \\ Mécanique des Matériaux, Téléport 2, 1 avenue Clément Ader, BP. 40109, 86961 Futuroscope \\ Chasseneuil Cedex, France \\ ${ }^{2}$ Airbus, Saint Martin du Touch, France
}

\begin{abstract}
The influence of casting defects on fatigue strength of an investment cast Ti$6 \mathrm{Al}-4 \mathrm{~V}$ alloy is investigated. The most common of these defects are: pinhole, linear defect and inclusion. Each of them is currently defined by its size, morphology and position from the surface but is different from each other for a same type. An experimental campaign is defined with different types of defect. The first part of the campaign is focused on the influence of an artificial and spherical defect, considering two different surface conditions. It is shown that fatigue behaviour of this alloy is very sensitive to the surface condition of this artificial defect despite stress concentrations at the tip of the defect. The second part of the campaign is focused on casting defects: reduction of fatigue life is quantified and it is shown that an electro-discharge machined defect cannot be representative of pinhole.
\end{abstract}

\section{Introduction}

Casting defects are metallurgical heterogeneities creating during the casting process. Their influence on fatigue strength is widely studied for aluminium [1] and steels [2]. However, fewer results can be found for titanium alloys [3]. The aim of this paper is to analyze the influence of casting defects by both quantifying reduction of the fatigue strength because of these defects and determining the critical defect size under which fatigue strength is not affected.

\section{Material data, defects and fatigue tests}

Flat castings were manufactured with a thickness of $5 \mathrm{~mm}$. They are used for both determining reference fatigue properties and quantifying the reduction of fatigue strength due to defects.

In this study, defects are artificial or natural. Influence of artificial defects, which are electrodischarge machined (EDM), is studied according to two different surface conditions: an EDM surface which is characteristic of the machining method and a chemically milled (CM) surface which is characteristic of the chemical milling used to remove the scoured layer created during machining of defect by EDM. Natural defects are pinholes which were observed at the surface of flat castings.

This is an Open Access article distributed under the terms of the Creative Commons Attribution License 4.0, which permits unrestricted use, distribution, and reproduction in any medium, provided the original work is properly cited. 


\section{MATEC Web of Conferences}

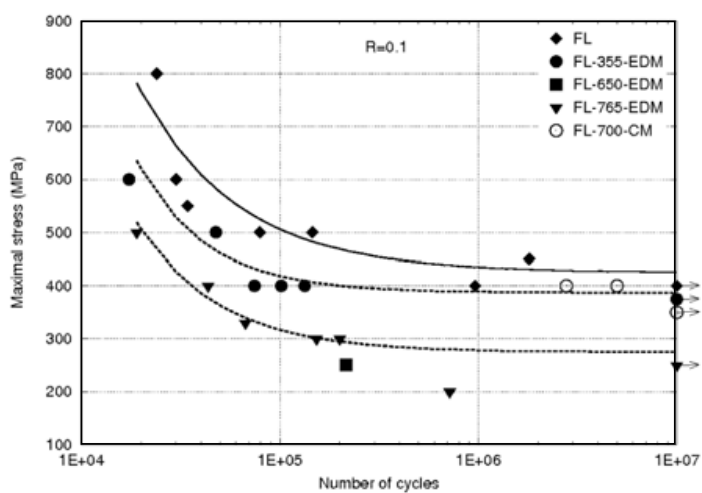

Figure 1. Influence of artificial defect.

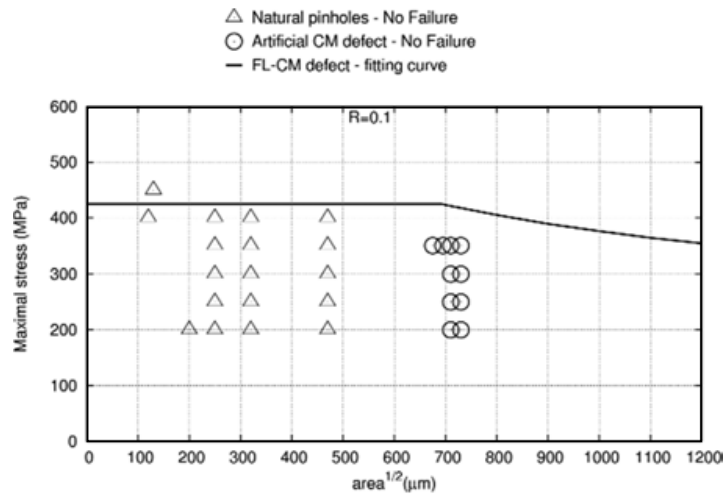

Figure 2. Kitagawa diagram at $10^{7}$ cycles with natural pinholes.

Fatigue tests are performed on flat specimens designed to be representative of aircraft components. Stress ratio is equal to 0.1 and fatigue limit is estimated thanks to a step loading procedure.

\section{Influence of artificial and natural defects}

Figure 1 presents SN curves for both types of artificial defects. Fatigue strength is reduced for all specimens containing EDM defects: there is a reduction of $40 \%$ for the EDM defect of $765 \mu \mathrm{m}$ at $10^{7}$ cycles. Considering a CM defect with a similar size, fatigue strength is not reduced.

Figure 2 presents Kitagawa diagram at $10^{7}$ cycles with natural pinholes and artificial CM defect. Pinholes observed at the specimen surface are not harmful because failure does not occur from these defects. This diagram shows that the critical defect size of pinhole is greater than or equal to $470 \mu \mathrm{m}$. Or, a $355 \mu \mathrm{m}$ EDM defect reduces fatigue strength of this material (Fig. 1).

\section{Conclusions}

The experimental campaign performed on the investment cast Ti-6Al-4V alloy shows that this material is very sensitive to the surface condition of artificial surface defects. Considering stress concentrations introduced by a surface defect is not sufficient to take account of its influence on fatigue strength of the 
FDMDII - JIP 2014

investment cast Ti-6Al-4V alloy. And so, an electro-discharge machined defect cannot be representative of pinholes.

\section{References}

[1] I. Koutiri, D. Belett, F. Morel, L. Augustin, J. Adrien, Int J Fatigue 47 (2013)

[2] L. Collini, A. Pirondi, R. Bianchi, M. Cova, P.P Milella, Procedia Engng 10 (2011)

[3] M. Filippini, S. Beretta, L. Patriarca, G. Pasquero and S. Sabbadini, Procedia Engng 10 (2011) 